\title{
Effect of Preventive and Curative Fungicide Treatment on Fusarium proliferatum Infected Maize - A Field Trial
}

\author{
TÜNDE PUSZTAHELYI ${ }^{1}{ }^{*}$, L. RADÓCZ ${ }^{2}$, CS. GELLÉRT ${ }^{1}$, SZILVIA KOVÁCS $^{1}$, \\ ZSUZSANNA SZABÓ ${ }^{3,4}$, I. PÓCSI $^{3}$ and A. VAD ${ }^{5}$ \\ ${ }^{1}$ Central Laboratory of Agricultural and Food Products, Faculty of Agricultural and Food Sciences and \\ Environmental Management, University of Debrecen, Böszörményi út 138, H-4032 Debrecen, Hungary \\ ${ }^{2}$ Faculty of Agricultural and Food Sciences and Environmental Management, Institute of Plant Protection, \\ University of Debrecen, Böszörményi út 138, H-4032 Debrecen, Hungary \\ ${ }^{3}$ Department of Biotechnology and Microbiology, Faculty of Science and Technology, \\ University of Debrecen, Egyetem tér 1, H-4032 Debrecen, Hungary \\ ${ }^{4} \mathrm{Ph}$.D. School of Biology, Szent István University, Páter Károly út, 1, H-2110 Gödöllő, Hungary \\ ${ }^{5}$ Institutes for Agricultural Research and Educational Farm, \\ University of Debrecen, Böszörményi út 138, H-4032 Debrecen, Hungary
}

(Received: 29 November 2016; accepted: 12 January 2017)

\begin{abstract}
There are extensive data on effects of antifungal agents on the plant pathogens, especially on Fusariums spp. species. However, investigations on the interaction of chemicals and the treated cultivars are rare. The aim of the study was to test two types of fungicide mixtures, azoxystrobin-propiconazole, and prothioconazole-tebuconazole, which are applied in wheat cultivars intensively, on six fodder maize hybrids that were infected with Fusarium proliferatum in the R1 growth stage in a field trial. The effect of the fungicide treatment was tested on the starch content and antifungal, antioxidant polyphenols of the kernels in the R3-R4 and R6 stage of the cultivars. The level of the fungal presence and the fumonisin concentration of the kernels were increased significantly under the artificial infection. The fumonisin concentration was variable at the R6 stage of the hybrid maize kernels. The treatment with prothioconazole and tebuconazole was found to be suitable when it was done before flowering, while the azoxystrobin-propiconazole treatments were equally successful before and after maize flowering considering the decreasing fumonisin concentration of the kernels. Both fungicide mixtures, when they were applied after maize flowering, affected the starch biosynthesis to the R3-R4 stage significantly. Meanwhile, azoxystrobin-propiconazole also significantly affected the antioxidant flavone/flavanol contents from the R3-R4 stage to the R6 stage.
\end{abstract}

Keywords: Fumonisin, Fusarium proliferatum, maize, fungicide, antioxidant.

Preharvest maize ear rot causes huge economic problem worldwide. Recently, Fusarium proliferatum, F. verticillioides and F. subglutinans (pink ear rot) (Logrieco et al., 1995) were found to be a potent causal agent of the kernel disease besides F. graminearum and F. culmorum (red ear rot) (Dorn et al., 2011). The fungal infection usually spread by insects, e.g. European Corn Borer (Ostrinia nubilalis Hübner) (Scarpino et al.,

* Corresponding author; e-mail: pusztahelyi@agr.unideb.hu 
2015), through the kernel injuries they cause. The fungal infection has an impact on the nutrient value of maize, and not only the starch content but the fatty acid composition can be affected (Dall'Asta et al., 2012).

Besides the decreased nutrient levels in the grains, the mycotoxin production of the fungal biomass is also a crucial problem and revealed in connections of all type of fungal infections (Nesic et al., 2014). Fusarium spp. are well known about the broad range of potentially toxic secondary metabolites, and $F$. proliferatum was reported as a potential producer of e.g. fumonisins, moniliformin (Scarpino et al., 2015), beauvericin (Moretti et al., 1994) and fusaproliferin (Logrieco et al., 2002). Therefore, prevention of the fungal infection and mycotoxin production in cultivars is a critical task.

A large set of metabolites in maize and cereals counteracts the fungal pathogens and Fusaria and reduces the mycotoxin accumulation in plants. These metabolites origin from the primary and the secondary metabolisms of plants and are very diverse. Amino acids, fatty acids, and their derivatives, carbohydrates, amines, terpenoids and phenylpropanoids are the best-known compounds (Gauthier et al., 2015). The last two groups with antioxidant properties are among the most frequently reported metabolites to take part in the plant defense mechanisms and some of them especially interfere with mycotoxin biosynthesis (Gauthier et al., 2015). Maize grains contain high level in total flavonoids followed by wheat, rice, and oat (Adom and Liu, 2002), and the most frequently cited flavonoids are the flavonols kaempferol and quercetin (Das and Singh, 2015).

Prevention against Fusarium ear rot relies on cultural practices to manage the disease: crop rotation, crop residue management, good nutrient supply (supplying high $\mathrm{N}$ and low K), control of the insect pests, and use of resistant hybrid plants. The relationships between the resistance and the mycotoxin production have been well documented (e.g. Pilu et al., 2011). The resistance to Fusarium spp. is quantitatively inherited, but, up to our knowledge, no fully resistant maize genotype has been discovered.

In the present work, two fungicide mixtures, azoxystrobin-propiconazole, and prothioconazole-tebuconazole were tested. Azoxystrobin (inhibits fungal respiration, Bartlett et al., 2002) and propiconazole (demethylation inhibitor; Taton et al., 1988) mixtures are recommended in maize cultivars against Aureobasidium zeae (eyespot) and Helminthosporium spp. (leaf blight) by the manufacturers. Meanwhile, prothioconazole and tebuconazole (dual demethylation inhibitor fungicide; Taton et al., 1988) are applied as mixture mainly on cereal cultivars. Interesting, that the fungitoxicity of these compounds is well understood, while the information on the effects on plant metabolism and plant-fungal interactions are rare (Wu and von Tiedemann, 2001; Blandino et al., 2012). Our aim was to highlight at least a part of the combined effect of the chemicals mentioned above on maize-Fusarium interaction. Therefore, the artificially infected maize hybrids were investigated under treatment at and after flowering. The rate of the fungal presence was characterized by visual evaluation and measurement of the total fumonisin content of the kernels in ELISA. The response to these specific fungicide treatments was evaluated by measuring the total antioxidant polyphenol, the flavone/flavonol content and storage nutrient starch levels. 


\section{Materials and Methods}

\section{Cultivation of maize hybrids}

The field experiment was conducted in the experimental areas of the Institute of Plant Protection, the University of Debrecen in 2015 using the following fodder maize hybrids: DKC5007, DKC5031, DKC4608 (Monsanto), and P9074, P9911, P0216 (DuPont Pioneer). The experimental design was a randomized complete block design (RCBD) with three blocks for each treatment. The research field composed of 108 squares, all of which had the length of $4 \mathrm{~m}$ and $2.25 \mathrm{~m}$ of width. In every square, there were three rows, the distance of the plants was applied as it was described for that hybrid by the producer. Seeds were planted approximately $5 \mathrm{~cm}$ deep. Plants were fertilized and frequently irrigated to avoid any nutrient deficiency or moisture stress.

The respective blocks of the hybrids were treated with a mixture of $125 \mathrm{~g}^{-1}$ prothioconazole and $125 \mathrm{~g} \mathrm{l}^{-1}$ tebuconazole and with a combination of $141.4 \mathrm{~g} \mathrm{l}^{-1}$ azoxystrobin and $122.4 \mathrm{~g} \mathrm{l}^{-1}$ propiconazole antifungal compounds both preventively and curatively. The chemicals were applied in $11 \mathrm{ha}^{-1}$ concentration in early flowering and after flowering of maize. The fields did not get any other treatment during the cultivation. All treatments of all hybrids were repeated three times.

\section{Contamination with Fusarium proliferatum}

F. proliferatum ITEM2287 strain was isolated from maize. The strain was inoculated on Czapek-Dox plates (HiMedia Laboratories) and incubated at $25^{\circ} \mathrm{C}$ for 7 days. The conidiospores were collected suspending them in distilled water and centrifuged three times at $1600 \mathrm{rpm}$ for $4 \mathrm{~min}$. The spore concentration was determined in a Bürker chamber, and a suspension was made in the final concentration of $10^{5} \mathrm{ml}^{-1}$ in sterile physiological salt solution and loaded into spreading flasks. The maize hybrids were contaminated in the R1 growth stage (Abendroth et al., 2011) of the plants by a nail punch (mimicking the damage by insects) at the uppermost $1 / 3$ of the ear through the husk leaves and spraying the conidiospores to the wound.

\section{Evaluation of the Fusarium contamination}

In the R6 stage of the maize cultures, the Fusarium infection was evaluated visually by the intensity of the fungal disease on the ears (Fischl, 1977). The contamination was rated and scored with 1-10 points as the following: (1) contaminated only at the ear tip; (2) 4-5 rows of the kernels at the tip; (3) diffusively at the 1/3 uppermost part of the ear; (4) up to the middle of the ear; (5) diffusively at the $2 / 3$ of the ear; (6) covering the $2 / 3$ of the upper part; (7) continuous mycelial cover on the $1 / 3$ of the ear; (8) constant mycelial cover on the $2 / 3$ of the ear; (9) total mycelial coverage; (10) totally damaged ear. The scores of the ears were summarized, and the average was taken for every block. 


\section{Sample taking and preparation}

Samples were taken at the R6 reproductive stage of the maize. The ears without the husk leaves were let air-dry for 2 days until further preparations. Then photos were taken on the samples, and the kernels were dried to steady weight in a drying cabinet. The dried seeds were ground and used in further analyses.

\section{Dry weight}

The dry weight of the kernels was determined by drying them in a $60{ }^{\circ} \mathrm{C}$ drying cabinet until a stable weight was reached. All measurements were done repetitively $(n=3)$. All analytical measurements have been related to the dry matter.

\section{Starch}

The starch content was measured based on the Hungarian standard MSZ 683018:1988. A portion of $2.5 \mathrm{~g}$ of ground sample was weighed in a $100 \mathrm{ml}$ Kohlrausch volumetric flask and mixed with $25 \mathrm{ml}$ of $0.31 \mathrm{M} \mathrm{HCl}$ solution. After addition of another $25 \mathrm{ml}$ of $0.31 \mathrm{M} \mathrm{HCl}$ solution, the suspension was heated on a boiling water bath for 15 min. Once the hydrolysis was finished, $20 \mathrm{ml}$ of cold distilled water was added. After fast cooling, clarification with $5 \mathrm{ml}$ of Carrez I and $5 \mathrm{ml}$ of Carrez II solutions was performed. Finally, the volumetric flask was filled up with distilled water, its content was properly mixed, and filtrated using a filtration funnel. The obtained filtrate was transferred to a polarization tube $(20 \mathrm{~cm})$ and measured using a polarimeter (Zeiss) at $20{ }^{\circ} \mathrm{C}$ with Na-lamp. The starch content related to dry weight was calculated as the following:

$$
[(\alpha \times 1000) /(184.6 \times 20 \mathrm{~cm} \times 2.5 \mathrm{~g})]^{*}[100 /(100-\mathrm{N})]=\operatorname{starch} \%
$$

$\alpha$, the angle of rotation; $\mathrm{N}$, water content.

All measurements were repeated three times.

\section{Polyphenols and flavonoids (flavones/flavanols)}

The determination of total phenolic compounds was made with Folin-Ciocalteu method (Kaur and Kapoor, 2002). Briefly, $5 \mathrm{~g}$ of ground maize was made up to $50 \mathrm{ml}$ with methanol:water (80:20), mixed thoroughly for $30 \mathrm{~min}$ and filtered through filter paper (Whatman grade 292; Millipore). From the extract, $0.5 \mathrm{ml}$ was reacted with $2.5 \mathrm{ml}$ of Folin-Ciocalteu reagent and of $2 \mathrm{ml}$ of $20 \%$ (w/v) sodium carbonate. The mixture was let to stand for $2 \mathrm{~h}$ in the dark, and the absorbance was measured at $760 \mathrm{~nm}$. The total phenolic content was calculated from the calibration curve, and the results were expressed as mg of gallic acid equivalent (GAE) per $100 \mathrm{~g}$ dry weight.

The determination of flavone/flavanol compounds was made per the aluminum chloride colorimetric method of Chang et al. (2002). Briefly, $1 \mathrm{ml}$ of the extract was combined with $4 \mathrm{ml}$ of distilled water and then $0.3 \mathrm{ml}$ of $5 \% \mathrm{NaNO}_{2}$ solution; $0.3 \mathrm{ml}$ of 
$10 \% \mathrm{AlCl}_{3}$ solution was added after $5 \mathrm{~min}$ of incubation, and the mixture was allowed to stand for $6 \mathrm{~min}$. Then, $2 \mathrm{ml}$ of $1 \mathrm{M} \mathrm{NaOH}$ solution was added, and the final volume of the mixture was brought to $10 \mathrm{ml}$ with distilled water. The mixture was let to stand for $15 \mathrm{~min}$, and absorbance was measured at $510 \mathrm{~nm}$. The total flavone/flavanol content was calculated from a calibration curve, and the result was expressed as $g$ catechin equivalent (CE) per $100 \mathrm{~g}$ dry weight.

\section{ELISA of fumonisins}

Fumonisins were measured with Fumonisin from maize ELISA kit (Sigma-Aldrich). The sample preparation and the measurements were done as it was described by the manufacturer.

\section{Statistics}

The three repetitions of a treatment of all six hybrids $(n=18)$ were evaluated in MS Office Excel program. Median values were calculated, and Student $t$-tests were performed. Significance was tested at the level of $p<0.05$ and $p<0.005$.

\section{Results}

The first task of the project was to get significantly infected cultivars and high fumonisin content in the maize kernels. The inoculation with F. proliferatum ITEM2287 strain was successful, as it was shown by the rate of the fungal presence that was evalu-

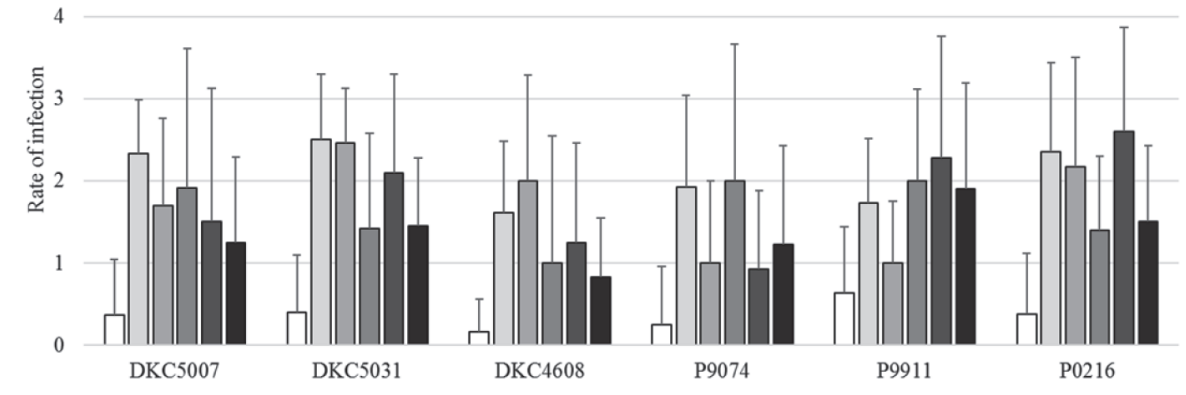

Fig. 1. Visual evaluation of Fusarium infection on the different hybrid maize DKC5007, DKC5031, DKC4608 (Monsanto), and P9074, P9911, P0216 (DuPont Pioneer) ears taken from the R6 stage. Mean and SD values were calculated from the data on three quadrats of each hybrids. The bars represent the mean from the different treatments in a sequence from left to right: non-infected and non-treated

controls, F. proliferatum treatment, prothioconazole-tebuconazole treatment before flowering, azoxystrobin-propiconazole treatment before flowering, prothioconazole-tebuconazole treatment after flowering, azoxystrobin-propiconazole treatment after flowering 
ated visually on the field (Fig. 1 and Fig. 2A) and by the fumonisin content (Fig. 2B). The prothioconazole-tebuconazole treatment was not successful in some hybrids (DKC4608, DKC5031, P0216) (Fig. 1) and caused only weak changes in the infection levels $(p<0.5)$ in comparison with the $F$. proliferatum infected but untreated cultivars (Fig. 2A). While, in most hybrids, azoxystrobin-propiconazole treatment decreased the visual signs of the infection (Fig. 1). Investigation of the R3 and R4 stage samples revealed fumonisin content under detection limit, however, in the R6 stage kernels, the ELISA measured high concentrations (within $0.05-65 \mathrm{mg} \mathrm{kg}^{-1}$ ) of the toxin. In the non-infected and non-treated controls, the Fusarium infection was observable only where the insect larvae damaged the ears; however, no significant fumonisin production was detected (Fig. 2B). In the presence of azoxystrobin and propiconazole fungicide, both the infection (Fig. 2A) $(p<0.005)$ and the fumonisin concentration (Fig. 2B) $(p<0.05)$ decreased significantly. While treatment with the prothioconazole-tebuconazole mixture had an inhibitory effect on the fumonisin content (Fig. 2B), when they applied before maize flowering, the infection rate was not reduced significantly by this mixture (Fig. 2A). The antioxidant polyphenol con-

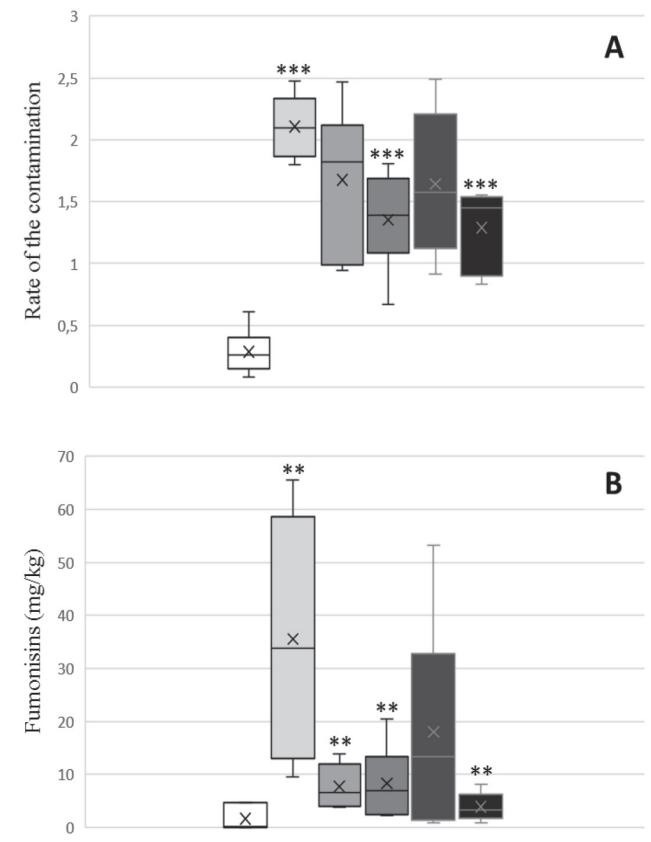

Fig. 2. The statistical analysis of the Fusarium infection (A) and the assay of the mycotoxin fumonisin (B) of the different hybrid maize DKC5007, DKC5031, DKC4608 (Monsanto), and P9074, P9911, P0216 (DuPont Pioneer) ears taken from the R6 stage. The boxplots represent the data from the different treatments in a sequence from left to right: non-infected and non-treated controls, F. proliferatum treatment, prothioconazole-tebuconazole treatment before flowering, azoxystrobin-propiconazole treatment before flowering, prothioconazole-tebuconazole treatment after flowering, azoxystrobinpropiconazole treatment after flowering. The boxplots contain the median (x) and the upper and lower quartiles. There were no outlier data. The significance of the differences at levels $p<0.05(* *), p<0.005$ $(* * *)$ was presented 
tent of the kernels as the respond to the fungal infection was tested in R3-R4 (Fig. 3A), and R6 (Fig. 3B) stages. Interestingly, in contrast to the flavone/flavanol content (Fig. 3C and D), the deviation of the measured polyphenol concentrations was high in the samples from the R3-R4 stage (Fig. 3A), while the values were stabilized in the R6 stage kernels (Fig. 3B). The flavone/flavanol content was low in R3-R4 stage (Fig. 3C) of the cultivars, and the fungicide treatments did not result in significant changes. Later, in the R6 stage, the flavone/flavanol values were increased and had higher deviations (Fig. 3D). Surprisingly, the azoxystrobin and propiconazole fungicide caused significant change when applied after flowering as a decreased flavone/flavanol content $(p<0.005)$ of the kernels was detected. We also tested the starch content (Fig. 4) and found significant differences $(p<0.005)$ in cultivars treated after flowering with any fungicide mixtures. The starch biosynthesis was more intensive in the R3-R4 stage hybrids treated by one of the fungicide mixtures (Fig. 4A), while, in the R6 stage (Fig. 4B), the difference was insignificant.

It was concluded that the effect of the fungicide mixtures, besides the applied chemical, strongly depended on the timing of the treatment. Only azoxystrobin and prop-
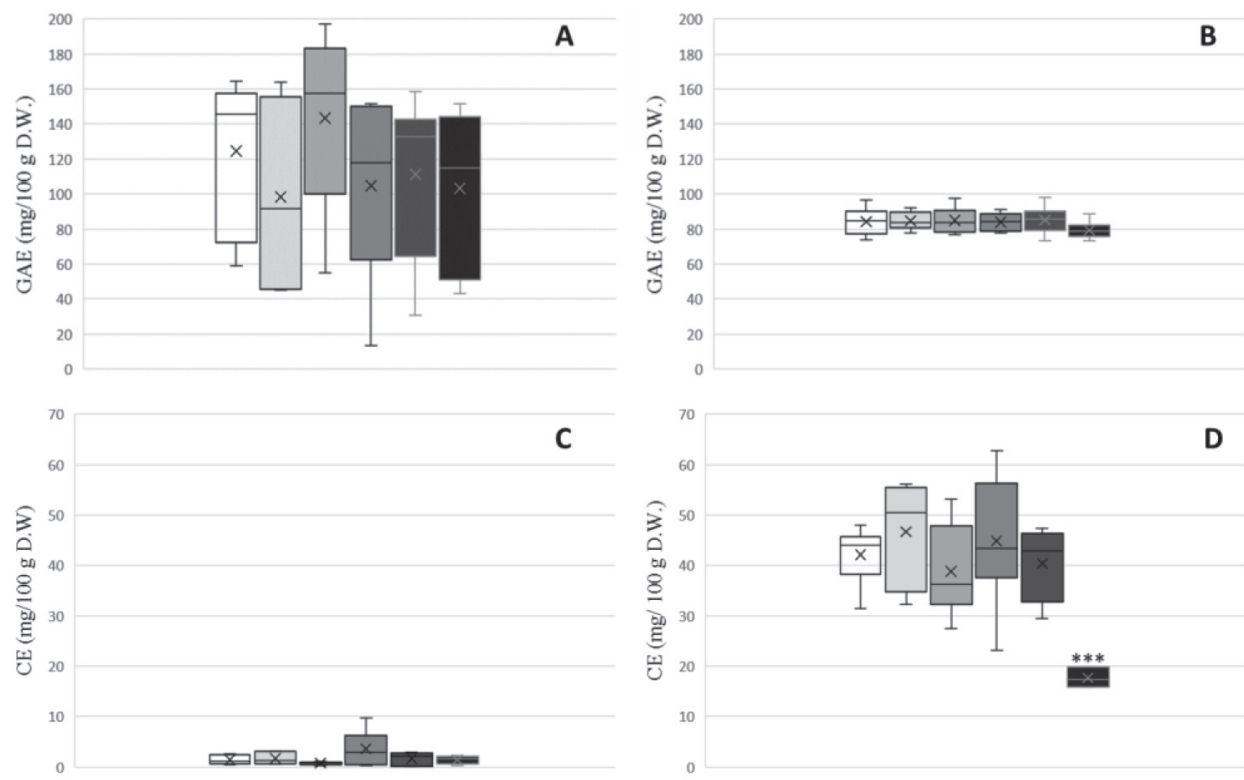

Fig. 3. Antioxidant total polyphenol (A-B) and flavonoids (flavones/flavanols) (C-D) contents of the different hybrid maize: DKC5007, DKC5031, DKC4608 (Monsanto), and P9074, P9911, P0216 (DuPont Pioneer) kernels taken from the R3-R4 stage (A and C) and the R6 (B and D) stage. The boxplot represents the data from the different treatments in a sequence from left to right: non-infected and non-treated controls, $F$. proliferatum treatment, prothioconazole-tebuconazole treatment before flowering, azoxystrobin-propiconazole treatment before flowering, prothioconazole-tebuconazole treatment after flowering, azoxystrobin-propiconazole treatment after flowering. The boxplots contain the median (x) and the upper and lower quartiles. There were no outlier data. The significance of the differences at level $p<0.005$ (***) was presented 

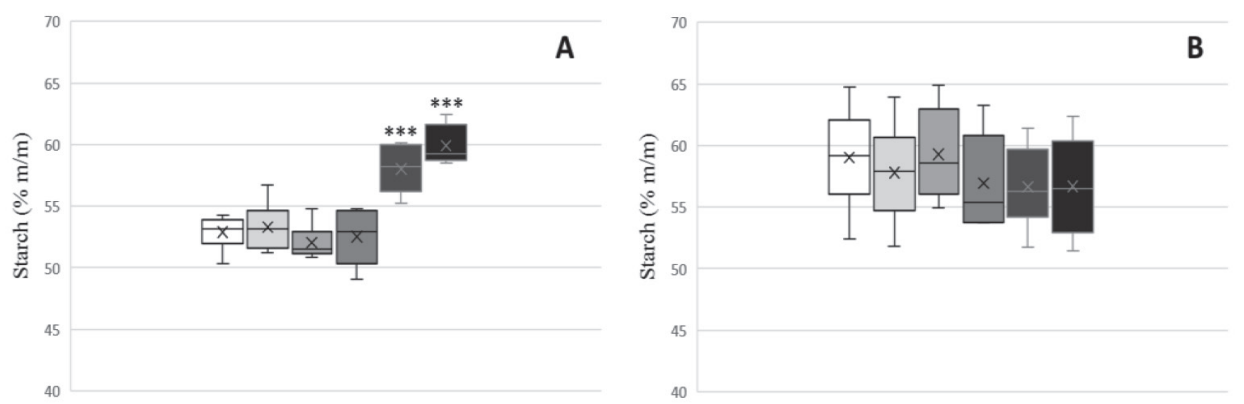

Fig. 4. Starch content of the hybrid maize DKC5007, DKC5031, DKC4608 (Monsanto), and P9074, P9911, P0216 (DuPont Pioneer) kernels taken from the R3-R4 stage (A) and the R6 (B) stage. The boxplot represents the data from the different treatments in a sequence from left to right: non-infected and non-treated controls, $F$. proliferatum treatment, prothioconazole-tebuconazole treatment before flowering, azoxystrobin-propiconazole treatment before flowering, prothioconazole- tebuconazole treatment after flowering, azoxystrobin-propiconazole treatment after flowering. The boxplots contain the median $(\mathrm{x})$ and the upper and lower quartiles. There were no outlier data. The significance of the differences at level $p<0.005(* * *)$ was presented

iconazole together caused significant changes in both the flavone/flavanol and fumonisin values at $p<0.005$ and $p<0.05$ significance levels, respectively.

\section{Discussion}

Recently, the injection of ears through husk leaves with a Fusarium conidiospore suspension was shown to be more efficient than stalk injection or soil contamination in inducing systemic and seed infection (Al-Juboory and Juber, 2013). Therefore, here the spore suspension also was applied spiking through the husk leaves. The results revealed that the applied inoculation method was efficient in causing infection with significant $(p<0.005)$ differences compared with non-infected and non-treated controls. It also has been demonstrated recently, that the dent stage (R5) of maize (Abendroth et al., 2011), with increasing starch content and reduced moisture content, was the most conducive for fumonisin production. Here, the acidic state of kernel induced more FB1 production (Picot et al., 2011). Accordingly, no fumonisin production was detected in the kernels of the R3, and R4 stage but in the R6 stage.

Generally, both fungicide mixture treatments were successful against $F$. proliferatum, but the timing of the treatment was highly critical not only from the point of the infection but the chemical composition of the kernels. It was concluded that prothioconazole and tebuconazole were more efficient when used preventively, while, azoxystrobin and propiconazole were more effective when they were applied after flowering.

Demethylation inhibitor fungicides, in addition to providing disease control, and particularly propiconazole and the quinone outside inhibitor fungicide strobilurins, have been shown to induce physiological benefits for plants (e.g. improved stalk strength, longer preserved green leaf tissue and delayed plant senescence) (Wu and von Tiede- 
mann, 2001). Blandino et al. (2012) found an increased photosynthetic activity in maize; however, no significant change in fumonisin content was detected when azoxystrobin and propiconazole were applied on flowering plants. No another physiological effect of the treatment has been demonstrated. The significantly decreased flavone/flavanol content as a new information made us careful for the timing of the treatment. Moreover, the fungal incidence was only decreased on plants but not dismissed by the azoxystrobin-propiconazole treatment when applied after flowering.

The presumable cause of the reduced flavone/flavanol content was the plant defense mechanisms as it was concluded in the case of winter wheat (Buśko et al., 2014). Alternatively, the flavonoids took part in the reaction to quinine inhibitor and the reduction of the level of FB1 in the pericarp (Pilu et al., 2011). Moreover, other polyphenols were increased in their concentration at the same time as the total polyphenol concentration did not change significantly. By our knowledge, there is no information on the precise mechanism, so it needs further research to elucidate the physiological process.

Although the azoxystrobin-propiconazole treatment generally was less effective against the Fusarium contamination and FB1 production when it was applied before flowering, in that case, it did not cause significant changes in the maize kernel composition; therefore, the preventive treatment is more recommended.

\section{Conflict of interests}

The authors declare no conflict of interest.

\section{Acknowledgements}

The authors thank the technical assistance of the students (Borbála Vágó, Fruzsina Matolcsi) worked in the maize fields. The authors also thank Agri-Corn Ltd. (Monostorpályi, Hungary) for the hybrid maize grains.

\section{Literature}

Abendroth, L. J., Elmore, R. W., Boyer, M. J. and Marlay, S. K. (2011): Corn growth and development. PMR 1 009. Iowa State University Extension, Ames, Iowa.

Adom, K. K. and Liu, R. H. (2002): Antioxidant activity of grains. J. Agric. Food Chem. 50, 6182-6187. doi: 10.1021/jf0205099

Al-Juboory, H. H. and Juber, K. S. (2013): Efficiency of some inoculation methods of Fusarium proliferatum and $F$. verticilloides on the systemic infection and seed transmission on maize under field conditions. Agric. Biol. J. North Am. 4, 583-589. doi: 10.5251/abjna.2013.4.6.583.589

Bartlett, D. W., Clough, J. M., Godwin, J. R., Hall, A. A., Hamer, M. and Parr-Dobrzanski, B. (2002): The strobilurin fungicides. Pest Management Sci. 58, 649-651.

Blandino, M., Galeazzi, M., Savoia, W. and Reyneri, A. (2012): Timing of azoxystrobin+propiconazole application on maize to control northern corn leaf blight and maximize grain yield. F. Crop Res. 139, 20-29. doi: 10.1016/j.fcr.2012.09.014

Buśko, M., Góral, T., Ostrowska, A., Matysiak, A., Walentyn-Góral, D. and Perkowski, J. (2014): The Effect of Fusarium inoculation and fungicide application on concentrations of flavonoids (apigenin, kaempferol, luteolin, naringenin, quercetin, rutin, vitexin) in winter wheat cultivars. Am. J. Plant Sci. 5, 3727-3736. doi: 10.4236/ajps.2014.525389 
Chang, C., Yang, M., Wen, H. and Chern, J. (2002): Estimation of total flavonoid content in propolis by two complementary colorimetric methods. J. Food Drug Anal. 10, 178-182.

Dall'Asta, C., Falavigna, C., Galaverna, G. and Battilani, P. (2012): Role of maize hybrids and their chemical composition in Fusarium infection and fumonisin production. J. Agric. Food Chem. 60, 3800-3808 dx. doi.org/10.1021/jf300250z

Das, A. K. and Singh, V. (2015): Antioxidative free and bound phenolic constituents in pericarp, germ and endosperm of Indian dent (Zea mays var. indentata) and flint (Zea mays var. indurata) maize. J. Funct. Foods 13, 363-374.

Dorn, B., Forrer, H. R., Jenny, E., Wettstein, F. E., Bucheli, T. D. and Vogelgsang, S. (2011): Fusarium species complex and mycotoxins in grain maize from maize hybrid trials and from grower's fields. J. Appl. Microbiol. 111, 693-706. doi: 10.1111/j.1365-2672.2011.05091.x

Fischl, G. (1977): A kukorica fuzáriumos csőfertőzöttsége és csírázási \%-a közötti összefüggés vizsgálata. (Study of the correlation between Fusarium ear rot and the sprouting ratio of maize.) Növényvédelem 13, 446-448. (in Hung.).

Gauthier, L., Atanasova-Penichon, V., Chéreau, S. and Richard-Forget, F. (2015): Metabolomics to decipher the chemical defense of cereals against Fusarium graminearum and deoxynivalenol accumulation. Int. J. Mol. Sci. 16, 24839-24872. doi: 10.3390/ijms161024839

Kaur, C. and Kapoor, H. C. (2002): Anti-oxidant activity and total phenolic content of some Asian vegetables. Int. J. Food Sci. Technol. 37, 153-161.

Logrieco, A., Moretti, A., Ritieni, A., Bottalico, A. and Corda, P. (1995): Occurrence and toxigenecity of Fusarium proliferatum from preharvest maize ear rot, and associated mycotoxins, in Italy. Plant Dis. 79, 727-731.

Logrieco, A., Mul`e, G., Moretti, A. and Bottalico, A. (2002): Toxigenic Fusarium species and mycotoxins associated with maize ear rot in Europe. European J. Plant Pathol. 108, 597-609.

Moretti, A., Logrieco, A., Bottalico, A., Ritieni, A. and Randazzo, G. (1994): Production of beauvericin by Fusarium proliferatum from maize in Italy. Mycotoxin Res. 10, 73-78. doi: 10.1007/BF03192255.

Nesic, K., Ivanovic, S. and Nesic, V. (2014): Fusarial toxins: secondary metabolites of Fusarium fungi. Rev. Environ. Contam. Toxicol., 228, 101-120.

Picot, A., Barreau, C., Pinson-Gadais, L., Piraux, F., Caron, D., Lannou, C. and Richard-Forget, F. (2011): The dent stage of maize kernels is the most conducive for fumonisin biosynthesis under field conditions. Appl. Environ. Microbiol. 77, 8382-8290. doi: 10.1128/AEM.05216-11

Pilu, R., Cassani, E., Sirizzotti, A., Petroni, K. and Tonelli, C. (2011): Effect of flavonoid pigments on the accumulation of fumonisin B1 in the maize kernel. J. Appl. Genet. 52, 145-152. doi: 10.1007/s13353010-0014-0

Scarpino, V., Reyneri, A., Vanara, F., Scopel, C., Causin, R. and Blandino, M. (2015): Relationship between European Corn Borer injury, Fusarium proliferatum and F. subglutinans infection and moniliformin contamination in maize. F. Crop Res. 183, 69-78. doi: 10.1016/j.fcr.2015.07.014

Taton, M., Ullmann, P., Benveniste and P., Rahier, A. (1988): Interaction of triazole fungicides and plant growth regulators with microsomal cytochrome P-450-dependent obtusifoliol 14 $\alpha$-methyl demethylase. Pestic. Biochem. Physiol. 30, 178-189. doi:10.1016/0048-3575(88)90051-X

Wu, Y.-X. and von Tiedemann, A. (2001): Physiological effects of azoxystrobin and epoxiconazole on senescence and the oxidative status of wheat. Pestic. Biochem. Physiol. 71, 1-10. doi: 10.1006/pest.2001.2561 